\title{
Evaluation inertielle : état de la question et perspectives
}

Inertial assessment: current knowledge and perspectives

JIDOVTSEFF B, CROISIER JL, DEMOULIN C, CRIELAARD JM.

Sci Sport 2008; 20:304-307 


\section{Résumé}

Objectifs - établir un état des lieux de l'évaluation musculaire inertielle Actualités - l'évaluation inertielle peut se réaliser avec un simple matériel de musculation. L'approche dynamométrique, plus précise, connaît un intérêt croissant dans le milieu sportif. En évaluant la performance musculaire au moyen de charges sous-maximales, elle permet d'établir des profils charge-vitessepuissance, d'étudier la fatigabilité musculaire ou encore d'analyser la cinétique des mouvements. Une rigueur méthodologique apparaît indispensable afin de garantir la fiabilité et la reproductibilité des résultats.

Perspectives et projets - les perspectives d'utilisation s'avèrent nombreuses dans le domaine sportif et pourraient s'étendre à certains contextes cliniques. La revue de la littérature indique la nécessité d'une clarification des recommandations et des protocoles d'évaluation.

Conclusions - l'évaluation dynamométrique inertielle constitue une méthode prometteuse, qui pourrait révolutionner l'évaluation musculaire sur le terrain.

Mots clés : évaluation, muscle, inertiel, dynamomètre, force-vitesse-puissance

\section{Abstract}

Aims - to present the current status of the inertial muscular assessment

Current knowledge - this method stays feasible with very basic resistance training materials. The dynamometric approach, more accurate, presents growing interest in the sport context. Inertial assessment with sub-maximal loads allows the description of force-power-velocity relationships, the investigation of 
muscular fatigue or the kinematical analysis of movement. Rigorous protocols are requested in order to warrant the results reliability.

Points of view and plans - clarification of the recommendations to the users and of the protocol designing process seems indispensable. Perspectives of use appear numerous in sport pursuit, and may be interesting in some clinical contexts.

Conclusion - The inertial dynamometric assessment appears like an accessible method which may revolutionize field muscular assessment.

Key words: assessment, muscle, inertial, dynamometer, force-velocity-power

\section{Introduction}

Les professionnels du sport se trouvent actuellement confrontés à de nombreuses techniques d'évaluation musculaire présentant des caractéristiques techniques et biomécaniques très spécifiques $[3,57,70]$. Les différences se marquent essentiellement au niveau du type de mouvement (mouvement analytique versus mouvement complexe), ainsi qu'au niveau du mode et de la cinétique de contraction (isométrique, isocinétique, inertielle). On ne peut 
affirmer qu'une technique soit meilleure qu'une autre. Son choix dépendra de l'objectif recherché, du contexte d'évaluation, mais aussi de l'accessibilité du matériel.

La plupart des activités quotidiennes et sportives se réalisent sous l'influence du champ de gravité terrestre qui définit les lois de la dynamique. Les mouvements impliquent l'accélération et la décélération de masses constantes, et engendrent le développement de forces inertielles. Compte tenu du principe de spécificité, l'évaluation inertielle devrait constituer un instrument de référence, en particulier si l'on souhaite que le mouvement d'évaluation se rapproche des gestes d'entraînement ou de la vie quotidienne.

Le concept de l'évaluation inertielle n'est pas clairement défini dans la littérature et reprend une multitude d'exercices musculaires dynamiques réalisés contre le poids du corps ou contre une résistance de masse constante. Dans tous les cas, le travail musculaire s'oppose à la gravité. Dans la littérature, ces exercices sont régulièrement qualifiés $d^{\prime}$ « isotonique », $d^{\prime}$ « iso-inertiel » ou de « balistique ». Le terme isotonique, signifiant que la tension musculaire reste constante au cours de l'effort, semble inadéquat dans la mesure où la force requise pour soulever une charge se modifie tout au long du mouvement $[3,9]$. Le terme isoinertiel, très utilisé actuellement [2-3, 28-29, 43], serait inapproprié dans la mesure ou l'inertie ne reste pas constante au cours du mouvement. Le terme inertiel apparaît plus adéquat car il fait référence à un effort qui modifie l'état de repos ou de mouvement d'un corps. On pourrait ainsi définir l'évaluation inertielle comme la quantification d'un exercice dynamique réalisé contre une charge de masse constante. Les exercices balistiques, quant à eux, se rapportent aux 
actions musculaires aboutissant à la projection d'un objet ou à l'autoprojection et constituent en fait une sous-catégorie des efforts inertiels.

La modalité d'évaluation inertielle s'avère indiscutablement avantageuse dans de nombreux contextes sportifs, mais aussi cliniques. Les mouvements sont généralement fonctionnels: ils reprennent les exercices classiques de musculation (le squat, la presse, les mouvements haltérophiles, le développé couché, etc.), les sauts verticaux, mais aussi des mouvements de tous les jours comme la mobilisation de boites lestées. Par ailleurs ces efforts, réalisés contre la gravité, reproduisent les accélérations et décélérations segmentaires naturelles.

Une évaluation musculaire se rapprochant des gestes quotidiens et sportifs sollicite une coordination intra- et inter-musculaire spécifique $[17,69]$. Il s'agit là d'un critère essentiel étant donné que la capacité d'une évaluation musculaire à refléter le niveau de performance est d'autant plus importante que les conditions d'effort se rapprochent de l'exercice fonctionnel [45].

\section{Les procédés simples de l'évaluation inertielle}

Avec un matériel simple, commun et peu coûteux, il est possible de réaliser une évaluation inertielle donnant des informations sur la force, la vitesse, la détente ou encore l'endurance locale.

\section{Evaluation de la force}

L'évaluation inertielle la plus simple et la plus courante consiste à apprécier la charge la plus élevée qu'un individu est capable de soulever à une seule reprise : il s'agit de la répétition maximale ou 1RM. Plusieurs méthodes ont été validées pour déterminer le $1 \mathrm{RM}[32,37]$. Les procédures se déroulent généralement en 
plusieurs étapes et aboutissent à la réalisation d'une seule répétition à proximité du maximum (tableau 1). Etant donné que la majorité des programmes de musculation se basent sur le $1 \mathrm{RM}$, sa détermination reste actuellement une démarche constructive dans le dosage de l'entraînement.

\section{Tableau 1. Etapes à suivre pour déterminer le 1RM}

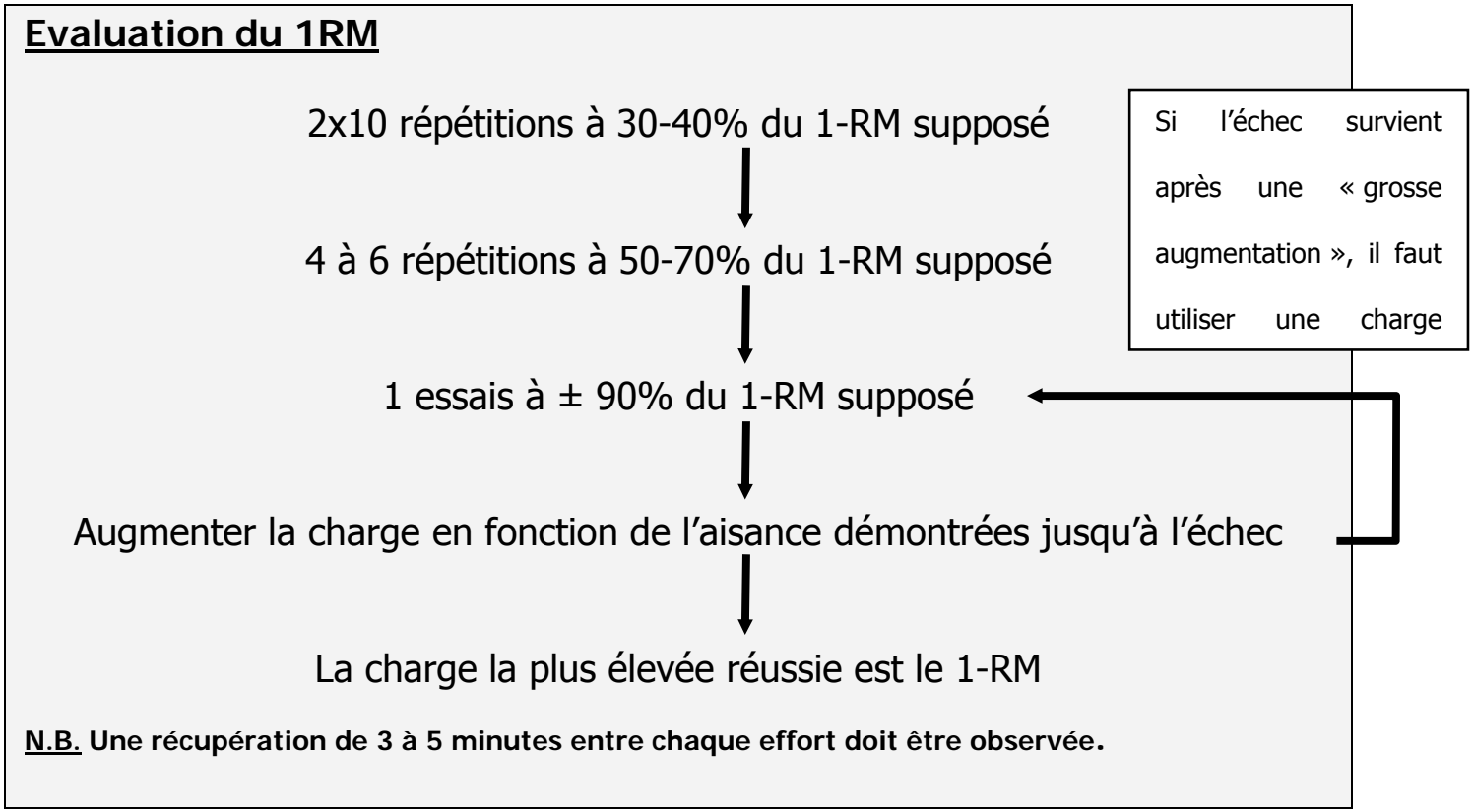

La détermination du 1 RM peut se réaliser sur toutes les machines et pour tous les mouvements (simples, complexes, linéaires ou angulaires) permettant la mobilisation de charges croissantes. Elle s'effectue sur le matériel utilisé à I'entraînement, ce qui lui confère une grande spécificité. Cependant, la charge maximale représente en réalité le niveau de force maximum qui peut être développée dans la partie la plus défavorable du mouvement concentrique [9, 39]. Une autre limite réside dans l'information fournie: si la performance représente le niveau de force maximale de l'individu, elle n'illustre en rien la puissance, ni la vitesse. 
La réalisation d'efforts extrêmement intenses reste déconseillée chez les sujets jeunes ou non initiés. Le risque lésionnel potentiel a justifié le développement de formules et de tables permettant l'estimation du 1RM à partir du nombre maximal de répétitions avec une charge inférieure (tableau 2) $[1,6,16,23,34$, $40-41,50]$. Le nombre de répétitions pour un pourcentage donné du 1RM pourrait cependant varier d'un exercice à l'autre [3, 36]. C'est donc avec prudence qu'il faudra interpréter et utiliser les résultats issus des ces méthodes d'extrapolation du 1RM.

Tableau 2 - Formules prédictives du 1RM à partir d’un nombre « $n$ » de répétitions réalisé avec une charge « $x »$.

\begin{tabular}{|l|c|}
\hline \multicolumn{1}{|c|}{ Auteurs } & Prédiction du 1RM \\
\hline Lander [34] & $\% 1 \mathrm{RM}=101,3-(2,67123 \cdot \mathrm{n})$ \\
Epley [16] & $1 \mathrm{RM}=0,033 \cdot \mathrm{n} \cdot \mathrm{x}+\mathrm{x}$ \\
O'Conner et al. [50] & $1 \mathrm{RM}=0,025 \cdot \mathrm{n} \cdot \mathrm{x}+\mathrm{x}$ \\
Mayhew et al. [40] & $1 \mathrm{RM}=\mathrm{x} \cdot\left(0,533+0,419 \mathrm{e}^{-0,055 \cdot \mathrm{n}}\right)^{-1}$ \\
\hline
\end{tabular}

\section{Les autres qualités musculaires}

L'évaluation inertielle ne se limite pas à la seule évaluation de la force musculaire. D'autres approches non dynamométriques permettent d'apprécier le dynamisme et l'endurance musculaire. Ainsi, les tests de détente verticale peuvent être assimilés à des épreuves inertielles car ils évaluent la capacité du sujet à mobiliser leur masse le plus haut possible. Les méthodes de Sargent, et d'Abalakov restent facilement réalisables sur le terrain [8]. Des systèmes plus sophistiqués (Ergojump ${ }^{\circledR}$ et Optojump ${ }^{\circledR}$ ) analysent la hauteur du saut et la qualité de l'impulsion en appréciant respectivement les temps de suspension et 
d'impulsion [8, 35]. Ils autorisent également la réalisation du drop jump (DJ) et de sauts enchaînés $[8,35]$. L'utilisation de formules offre une estimation de la puissance développée [10]. Ces épreuves, toujours limitées aux membres inférieurs, n’apprécient cependant jamais ni la vitesse gestuelle, ni la force maximale.

Pour apprécier les qualités de vitesse et de puissance musculaires dans un exercice précis sans dynamomètre, il faut répéter le plus vite possible le cycle entier du mouvement (montée et descente de la charge) avec une résistance très légère pour apprécier la vitesse (charge $<30 \%$ du $1 \mathrm{RM}$ ) ou moyenne pour apprécier la puissance (charge comprise entre 30 et $70 \%$ du 1RM). L'évaluation consiste simplement à chronométrer le temps mis pour réaliser un nombre précis de répétitions (entre 5 ou 10). Malheureusement, ce type d'évaluation s'accompagne d'un risque d'imprécision important. Une utilisation longitudinale exige de reproduire parfaitement la procédure d'un test à l'autre. L'amplitude du mouvement, qui doit être rigoureusement contrôlée, et le chronométrage manuel sont des sources d'erreur évidentes. La performance dépend de la vitesse moyenne développée sur tout le test, mais n'offre aucune information valide sur la vitesse réellement développée lors de chaque mouvement. Il en est de même pour la puissance. Par ailleurs, à mouvement égal, les sujets de petit gabarit sont inévitablement avantagés. Aussi, faudrait-il, pour réduire l'influence de la taille, rapporter la performance à l'amplitude totale du mouvement.

L'évaluation inertielle contribue également à apprécier l'endurance musculaire. La procédure classique consiste à compter le nombre maximum de répétitions réalisées avec une charge donnée, idéalement exprimée en pourcentage du 
maximum [52]. Une autre possibilité consiste à chronométrer le temps nécessaire pour réaliser un nombre important de répétitions avec une charge sousmaximale. Cette mesure simple ne permet jamais de quantifier le niveau de fatigue, ni l'évolution des paramètres de l'effort (force, vitesse, puissance) au cours des répétitions.

Affin d'explorer la fonction musculaire du rachis chez des sujets lombalgiques, certains auteurs ont élaboré des épreuves inertielles d'endurance musculaire, qui consistent à répéter plusieurs fois le soulèvement d'une charge dans des conditions très proches des gestes quotidiens [15, 38]. Le test PILE [38], par exemple, consiste à soulever une boite d'une masse constante jusqu'à une hauteur de $76 \mathrm{~cm}$. Un incrément de masse est proposé toutes les 20 secondes ( $+2,25 \mathrm{~kg}$ pour les femmes et $+4,5 \mathrm{~kg}$ pour les hommes). Le rythme de l'effort correspond approximativement à un soulevé toutes les 5 secondes. Le niveau de performance s'apprécie par la charge maximale soulevée.

L'évaluation musculaire inertielle peut donc tout à fait s'effectuer avec un matériel simple, accessible à tous. Les informations obtenues peuvent cependant manquer de validité et de précision. L'approche dynamométrique, certes moins accessible, offre en revanche une mesure précise de la performance musculaire lors d'exercices inertiels. Les perspectives d'utilisation de ces dynamomètres s'avèrent nombreuses à la fois dans le domaine de l'entraînement et en milieu clinique. 


\section{Evaluation inertielle dynamométrique}

L'évaluation inertielle dynamométrique utilise généralement des capteurs physiques pour mesurer les forces développées pendant un effort, mais aussi pour apprécier la vitesse produite et la puissance résultante $[7,20,29,43,61]$.

Les dynamomètres inertiels ne mesurent pas directement les forces produites par le sujet, mais plutôt les conséquences de celles-ci sur la mobilisation d'une charge. Ils utilisent généralement un capteur de déplacement et/ou un accéléromètre $[20,29,61]$. Le capteur de déplacement présente un câble que I'on attache à la charge, et qui s'enroule et se déroule au gré du mouvement. L'accéléromètre, de type piezorésistif ou capacitif, se positionne à I'horizontale ou à la verticale selon les modèles. Afin d'obtenir des mesures fiables, les capteurs physiques doivent être consciencieusement placés sur la barre ou sur le banc de musculation en respectant les consignes des constructeurs [30]. Pour garantir la validité des mesures, la charge devra être mobilisée linéairement. Une utilisation dans le cadre de mouvements complexes non guidés (développé couché, squat, arraché, ...) n'est envisageable que si le sujet testé maîtrise parfaitement la technique. La transportabilité de ces dynamomètres est incontestablement un avantage car elle autorise une évaluation musculaire rigoureuse sur le lieu de l'entraînement.

D’autres techniques, comme la caméra haute vitesse, l'analyse cinématique et les plates-formes de force, s'apparentent à une évaluation inertielle indirecte. Ces techniques onéreuses et d'utilisation complexe restent plus confinées à une utilisation de laboratoire. Elles sont surtout utilisées dans le cadre de l'analyse 
biomécanique des mouvements et demeurent moins adaptées à l'exploration des qualités musculaires.

\section{L'information dynamométrique}

Les capteurs utilisés par les dynamomètres inertiels mesurent directement soit le déplacement (capteur de déplacement), soit l'accélération (accéléromètre). La masse soulevée étant connue, toutes les autres grandeurs physiques s'obtiennent par traitement mathématique (figure 1). Un logiciel informatique d'analyse des signaux permet d'obtenir d'une part des paramètres chiffrés et d'autres part des courbes de mouvement.

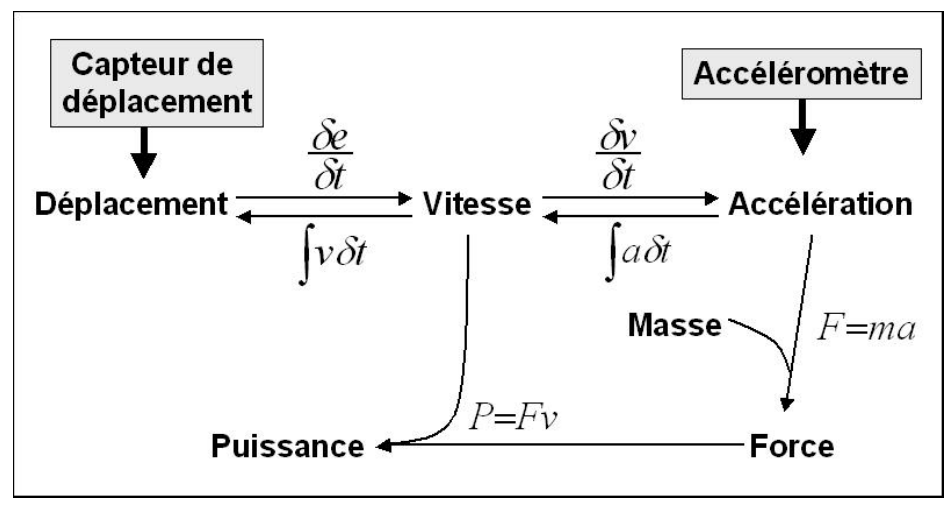

Figure 1. Traitement mathématique nécessaire pour obtenir les mesures de la force, de la vitesse et de la puissance à partir d'un capteur de déplacement ou d'un accéléromètre.

Les mesures les plus fréquentes concernent les valeurs moyennes et maximales de la vitesse, de la puissance ou encore de la force $[7,18,22,29,49,55]$. Le temps nécessaire pour atteindre la vitesse ou la puissance maximale est également utilisé comme paramètre de l'explosivité [22, 29]. L'évaluation inertielle permet également une analyse de courbe qui contribue à l'analyse qualitative des mouvements. 


\section{Possibilités de l'évaluation inertielle dynamométrique}

L'approche dynamométrique inertielle présente les qualités requises pour apprécier la puissance, la force, l'accélération, le déplacement ou encore le travail. Elle apparaît surtout comme la seule technique capable d'évaluer la vitesse, une qualité musculaire très importante, souvent négligée. Que ce soit sur le terrain ou en laboratoire, l'évaluation inertielle propose principalement trois champs d'investigation : les profils F-V-P, les épreuves de fatigue musculaire et I'analyse biomécanique des mouvements.

\section{Détermination des profils F-V-P}

L'établissement des relations F-V-P, requiert l'évaluation de la vitesse et de la puissance à différents niveaux de charge (figure 2). L'étude de la littérature révèle actuellement l'absence de consensus sur les protocoles. Les charges utilisées lors de l'évaluation inertielle peuvent être exprimées par rapport au poids du corps, par rapport au 1RM ou encore de manière absolue.

Certains auteurs réalisent leur évaluation avec des charges exprimées en pourcentage du poids corporel (\%PC) $[7,44]$. Si l'on accepte le principe selon lequel le niveau de force est proportionnel à la morphologie du sujet, cette méthodologie peut s'avérer intéressante. Elle reste malheureusement problématique pour des sujets hors normes. Par exemple, un sujet de $100 \mathrm{~kg}$ qui soulève $70 \mathrm{~kg}$ en développé couché devrait soulever des charges de 25, 50 et 75 kg s'il était évalué à 25,50 et $75 \%$ de son poids corporel. Un sujet pesant $80 \mathrm{~kg}$ et capable de mobiliser $120 \mathrm{~kg}$ serait, quant à lui et en suivant le même protocole, évalué à 20,40 et $60 \mathrm{~kg}$. 

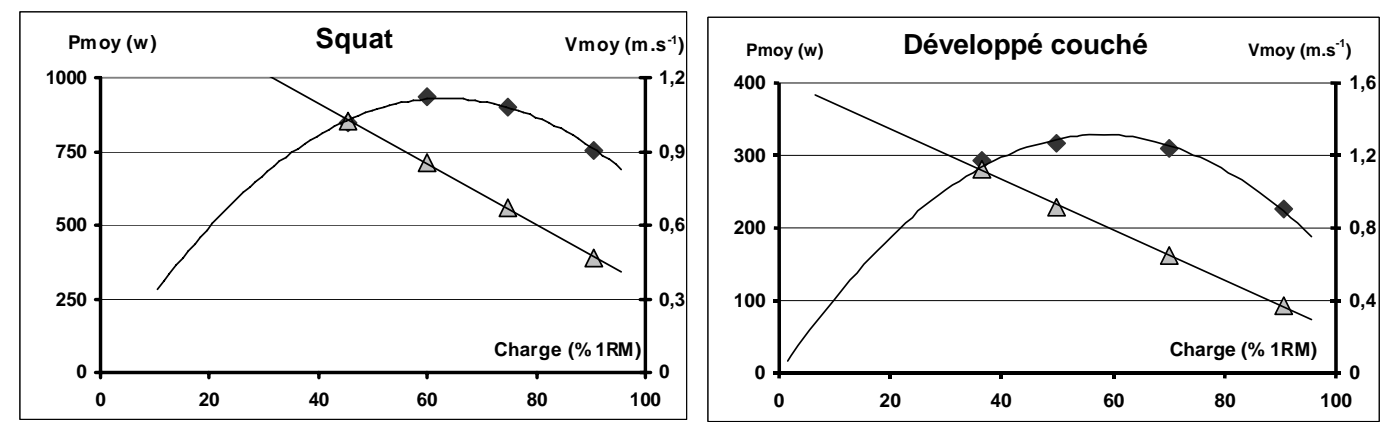

Figure 2 - Evolution de la vitesse (Vmoy) et de la puissance (Pmoy) en fonction de la charge relative (en \% du 1RM) lors de l'évaluation inertielle squat et du développé couché (d'après J idovtseff et al. [29]).

Plus généralement, on évalue tous les sujets avec des charges absolues préalablement déterminées $[4,19,55-56,58,63,65]$. L'augmentation de la charge se fait par paliers constants en fonction de l'exercice. Cette procédure reste facile à mettre sur pied lorsque plusieurs personnes sont évaluées en même temps. Deux approches sont possibles : la plus pratique consiste à utiliser les mêmes charges pour tous les sujets. Soit on utilise uniquement des charges mobilisables par tous et les plus forts restent alors explorés sur un profil incomplet, soit les sujets déplacent des charges tant qu'ils en sont capables et les plus forts réalisent un nombre plus important d'essais. L'autre solution consiste à proposer un accroissement de charge individualisé. Cette méthode permet de réaliser un nombre limité d'effort et donc un gain de temps, mais elle nécessite la connaissance préalable du niveau de force approximatif du sujet. L'établissement d'un protocole universel reste difficilement imaginable étant donné la diversité des performances musculaires pour un même mouvement. Cette méthode présente l'avantage d'établir un profil en une seule séance mais le risque d'un effet d'apprentissage est plus important. Pour limiter cet effet, il semble indispensable de réaliser, avant l'évaluation proprement dite, plusieurs répétitions de l'exercice en guise de familiarisation. Durant le test, l'expérimentateur sera attentif à l'évolution des résultats : des essais 
supplémentaires seront appliqués lorsque que la performance augmente entre les essais.

L'utilisation de charges relatives, exprimées en pourcentage de la charge maximale (\% 1RM) $[13,26,29,45,60-61,67]$, présente d'autres avantages : tout d'abord, tous les sujets exécutent un effort proportionnel à leur niveau de force; ensuite, cette modalité autorise les comparaisons inter-individuelles ; finalement, l'étude de la relation force-vitesse se réalise pour chaque sujet en utilisant le même nombre de charge. Cette procédure alourdit par contre le protocole ; en effet, le 1RM ne peut se déterminer le jour même de la séance d'évaluation inertielle, sous peine d'engendrer une fatigue préjudiciable. En conséquence, l'établissement d'un profil se réalisera en deux séances : la première pour déterminer le 1RM et une seconde pour l'évaluation inertielle. Si cette séance supplémentaire s'avère contraignante, elle permet cependant une bonne accoutumance avec le mouvement et avec les conditions expérimentales.

La séance d'accoutumance reste fortement conseillée lorsque le sujet n'est pas initié au mouvement test $[13,26,29,32,43,54]$. Il a été démontré dans d'autres contextes d'évaluation (isométrie et isocinétisme) que la performance peut s'améliorer de la première à la seconde séance par le seul apprentissage technique $[27,62,71]$. La séance de familiarisation réduirait effectivement cet effet d'apprentissage et serait d'autant plus nécessaire que le mouvement est complexe $[9,33]$. Elle devrait comprendre des conseils techniques et des séries de répétitions du mouvement test. Par ailleurs, elle est généralement mise à profit pour définir la position d'évaluation ainsi que les charges auxquelles les sujets seront testés. 
Le registre des charges relatives utilisées pour l'évaluation devrait idéalement explorer les trois zones critiques de la relation F-V-P. Les qualités de vitesse s'explorent au moyen de charges légères (entre 10 et $30 \%$ du $1 \mathrm{RM}$ ) alors que pour étudier la force, il convient d'utiliser des charges élevées (entre 80 et $100 \%$ du 1RM) $[30,45]$. Entre ces deux zones, on explore la puissance musculaire [12].

Lors de la mise en place d'un protocole, il importe de considérer le nombre de charges différentes et d'essais accordés. Afin d'écourter l'évaluation, il pourrait être proposé de ne réaliser qu'un seul essai à chaque charge. Cette approche reste critiquable, particulièrement dans le cadre de mouvements complexes. En effet, la réalisation d'un essai unique ne permet pas toujours l'obtention d'un résultat fiable. Une simple erreur technique influence la performance, et il apparaît plus judicieux d'accorder plusieurs essais à chaque charge en conservant le meilleur résultat. Afin de limiter le temps de l'évaluation et d'éviter toute accumulation de fatigue, nous conseillons néanmoins de ne pas dépasser 7 charges différentes et d'accorder au moins deux essais à chaque niveau de charge. Pour un même mouvement, le nombre total d'efforts devrait se limiter à une quinzaine. Lors d'un suivi longitudinal, le protocole pourra être adapté en fonction des résultats de la première séance test. Le nombre d'essais nécessaires diffère selon la charge. Aux charges légères trois ou quatre essais sont conseillés. Si la performance augmente significativement à chaque essai, l'évaluation doit être continuée à la même charge jusqu'à l'obtention d'une stabilisation de la performance. Dans ce cas précis, l'augmentation du nombre d'essais doit s'accompagner d'une augmentation de la récupération. Aux charges moyennes, deux à trois essais devraient suffire, pour deux essais seulement aux 
charges les plus élevées ( $\geq 80 \%$ du $1 \mathrm{RM}$ ). Certains auteurs retiennent la moyenne de tous les essais réalisés à une même charge [43]. Il nous semble plus adéquat de retenir le meilleur des différents essais réalisés à une charge donnée. Le mode de sélection n'est malheureusement pas souvent décrit dans la littérature, mais il semble que la vitesse maximale constitue un critère fiable compte tenu de sa reproductibilité et de sa sensibilité [30].

La récupération varie également : aux charges légères ( $<50 \%$ du $1 \mathrm{RM})$, un repos d'une minute s'avère suffisant, correspondant d'ailleurs à celui de l'évaluation isocinétique $[51,71]$, et à d'autres évaluations inertielles $[14,22$, 60]. Lors de tels efforts explosifs, la resynthèse du pool des phosphagènes se déroule en quelques dizaines de secondes [53]. Aux charges plus élevées, l'intensité et la durée de l'effort entraînent une fatigue plus prononcée justifiant une récupération de deux à trois minutes [29, 32, 45, 55, 67]. Une récupération écourtée pourrait altérer la performance et en conséquence la validité des mesures [22].

Les profils charge-vitesse et charge-puissance établis par ces protocoles s'avèrent d'une grande utilité dans le cadre du suivi de l'entraînement, mais aussi dans l'étude des caractéristiques musculaires. Une utilisation transversale permet de comparer des groupes ou des individus $[7,26,30]$. Une utilisation longitudinale met en évidence les effets spécifiques d'un entraînement [4, 19, 30, 47]. La détermination du 1RM n'est pas obligatoire, ce qui apparaît clairement avantageux dans de nombreux contextes, notamment cliniques. Les profils théoriques peuvent être mis à profit pour déterminer les charges d'entraînement 
à partir de critères précis de force, de vitesse et de puissance mais aussi pour estimer le 1RM.

Il faut noter que les paramètres maximaux n'évoluent pas de la même façon que les paramètres moyens. Pour le développé couché, on observe par exemple que la vitesse moyenne respecte une régression linéaire alors que la vitesse maximale diminue selon un équation polynomiale du second degré [28-29, 48]. Le profil hyperbolique de la puissance révèle également une évolution différente entre les valeurs moyennes et maximales. En fait, les paramètres maximaux ne considèrent que la partie la plus intense du mouvement, alors que les valeurs moyennes représentent le mouvement dans sa globalité. Récemment, il a été démontré que le choix du paramètre peut influencer l'interprétation des résultats, notamment lors du suivi de l'entraînement [30]. Pour être complète, I'analyse dynamométrique inertielle devrait envisager ces deux catégories de paramètres.

Par ailleurs, il faut souligner que l'exploration des profils force-vitesse-puissance dépend étroitement des possibilités des machines utilisées. En effet, les machines prévues pour subir des charges élevées sont de conception robuste et ne permettent généralement pas I'utilisation de charges très légères. Inversement, un matériel léger n'apparaît pas toujours adéquat pour accueillir des charges élevées. Dans un cas comme dans l'autre, le matériel pourrait limiter l'exploration de la fonction musculaire.

\section{Exploration de la résistance à la fatigue et de l'endurance musculaire.}

Dans certaines activités spécifiques, la performance dépend non seulement de la force et de la vitesse, mais aussi de la capacité à maintenir une intensité élevée pendant une période prolongée. Lorsque l'intensité de l'effort est sub-maximale, 
la sollicitation musculaire devient essentiellement anaérobie : on parlera de résistance à la fatigue. Dans le cas d'une intensité moyenne à faible, le métabolisme aérobie prédomine et l'on parle d'endurance musculaire.

Pour mesurer la fatigabilité dynamique des jambes, Bosco a proposé, il y a quelques années, des épreuves de sauts répétés de 30 à 60 secondes [8]. Lors de ces tests, la fatigue s'apprécie à travers la diminution de la détente verticale. Il s'agit là d'une des seules épreuves inertielles d'endurance musculaire rencontrée dans la littérature. Le matériel utilisé (tapis de Bosco) ne permet cependant pas une évaluation précise des qualités de vitesse et de puissance au cours de l'épreuve. La dynamométrie inertielle, en palliant cette lacune, offre de nouvelles perspectives d'exploration musculaire. Différents protocoles, inspirés de ce qui a été réalisé en isométrie et en isocinétisme, pourraient trouver des applications dans un contexte sportif et peut être même clinique.

L'endurance musculaire pourrait par exemple s'apprécier en maintenant une intensité d'effort constante (puissance cible) le plus longtemps possible avec une charge sous-maximale. Une autre possibilité d'épreuve consisterait à apprécier l'évolution des paramètres inertiels au cours d'un nombre standardisé de mouvements. Pour étudier le phénomène de fatigue, on pourrait également s'inspirer du test isométrique proposé par Vollestad [66], en réalisant de manière cyclique plusieurs répétitions sous-maximales, suivies d'une répétition à puissance maximale (Figure 3). L'endurance musculaire serait alors appréciée par la durée de l'effort et la diminution de la puissance lors des répétitions maximales. 


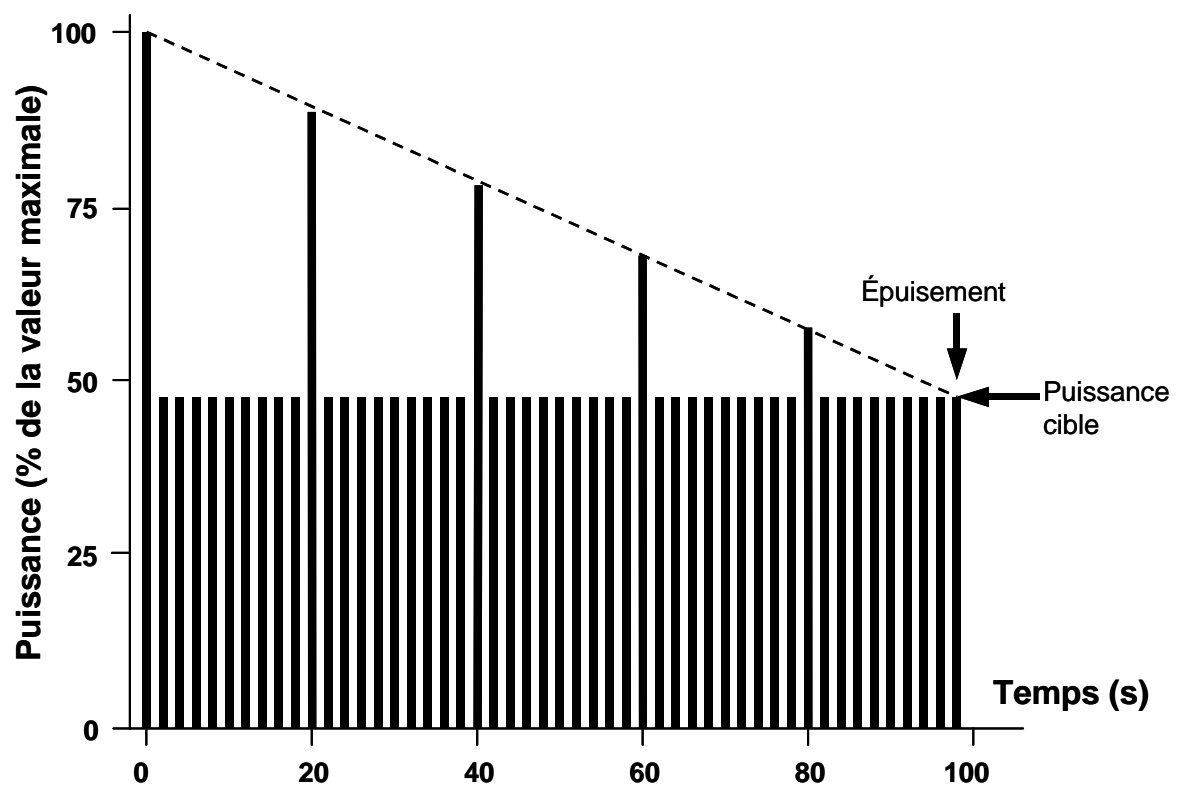

Figure 3 - Représentation schématique d'une épreuve d'endurance musculaire alternant des efforts maximaux avec des séries de répétitions sous-maximales (inspiré de Vollestad [66])

La durée et l'intensité (niveau de charge et rythme des mouvements) des efforts doivent évidemment être fixées en fonction des caractéristiques de la population étudiée. Lorsque l'on s'intéresse à l'endurance musculaire, la charge ne devrait pas dépasser 30 à $40 \%$ du maximum et l'effort devrait durer au moins une minute afin de garantir une participation importante du métabolisme aérobie. Par contre, les épreuves de résistance à la fatigue apparaissent plus courtes mais plus intenses afin de solliciter les filières anaérobies. Dans ce cas, et à l'instar d'un test de Wingate [24], nous proposons d'initier l'épreuve à une intensité maximale qui sera maintenue soit pendant un nombre fixe de répétitions, soit pendant un laps de temps défini. Cette façon de procéder, également courante en isocinétisme, apparaît plus facilement standardisable puisque le sujet ne doit pas contrôler son effort : son engagement est maximum dès le départ. Au cours de ces épreuves de fatigabilité initiées à intensité maximale, deux informations méritent une attention particulière : la diminution de la performance au cours de l'épreuve (exprimée par un index de fatigue) et l'intensité moyenne développée 
au cours de l'épreuve. Un test comportant trente répétitions maximales avec une charge relative de $40 \%$ du 1 RM a été mise au point avec succès dans le cadre du développé couché [30] (figure 4). Ce niveau de charge favorise, en début d'épreuve, le développement d'une puissance proche du maximum. Une charge élevée ou plus légère pourrait s'envisager si l'on souhaite explorer l'enduranceforce ou l'endurance-vitesse respectivement.

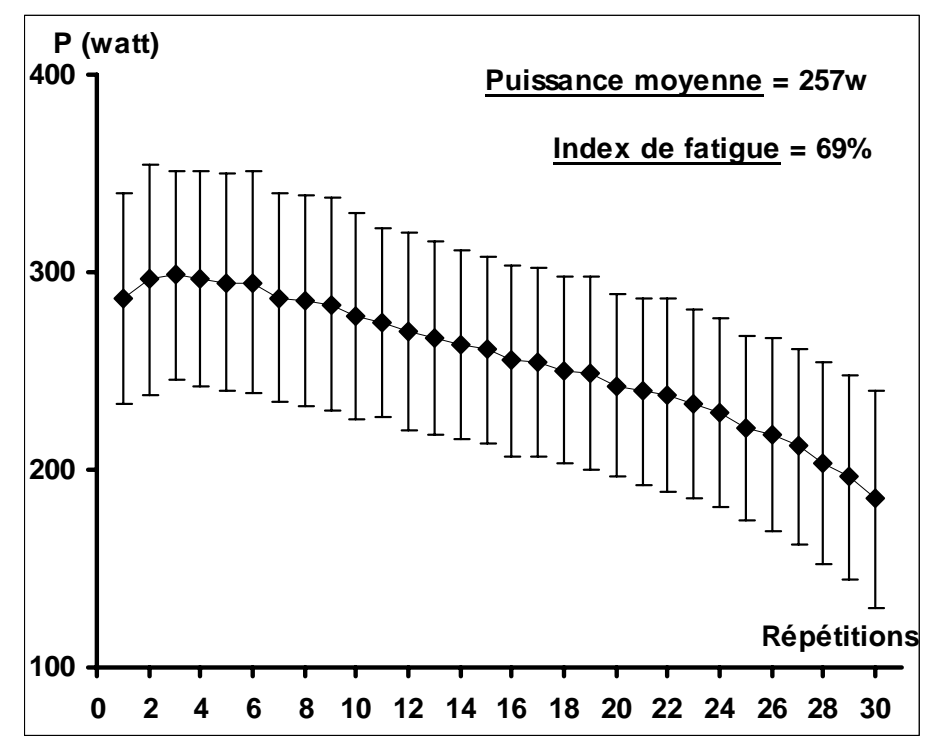

Figure 4 - Evolution de la puissance lors d'un épreuve de résistance à la fatigues ( 30 répétitions maximales çà $40 \%$ du 1RM) réalisée en développé couché [28].

Si la littérature actuelle consacrée à ce type d'évaluation apparaît particulièrement pauvre, les recherches futures s'attelleront à définir plus clairement les lignes de conduites à respecter, afin d'obtenir des épreuves de qualité.

\section{Analyse biomécanique des mouvements}

Les dynamomètres inertiels sont des outils pertinents pour l'analyse biomécanique de certains mouvements. Grâce à une mesure continue des paramètres cinétiques, ils donnent des informations concrètes sur certains paramètres de l'effort comme les actions musculaires propulsives et frénatrices, 
les phases d'accélération et de décélération, les impulsions mises en jeu, etc [30]. L'analyse des courbes de force, de vitesse et de puissance et de déplacement se justifie dans l'exploration biomécanique d'un mouvement particulier, mais aussi dans le suivi longitudinal d’un entraînement spécifique, ou d'une rééducation musculaire.

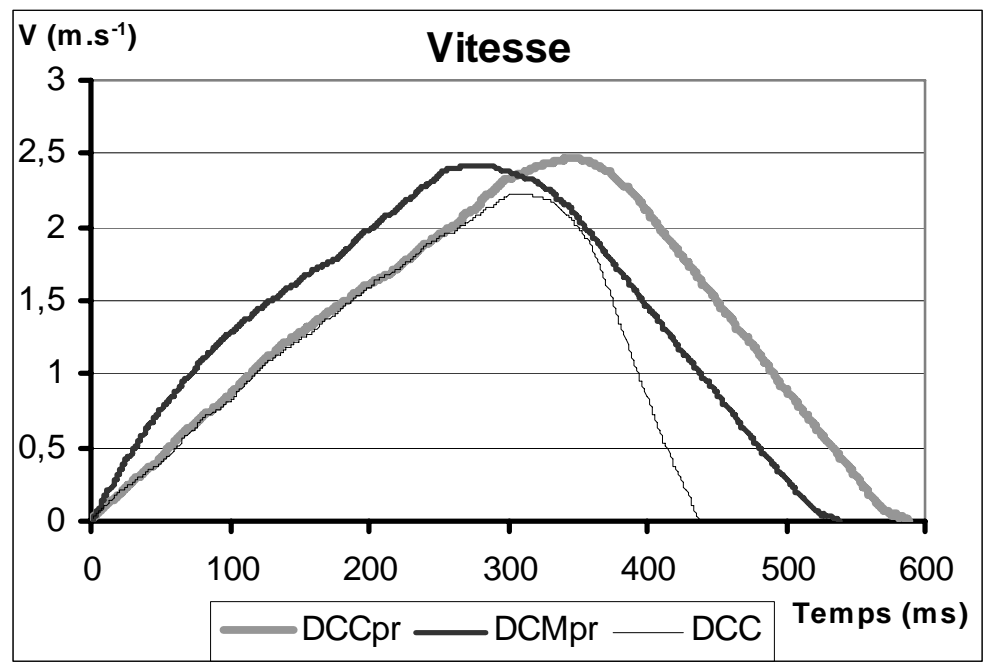

Figure 5 - Evolution de la vitesse au cour d'un développé couché réalisé par un même sujet selon trois modalités déférentes: concentrique (DCC), concentrique avec projection (DCCpr) et complet avec projection (DCMpr)

A titre d'exemple, l'évaluation dynamométrique inertielle à été utilisée afin d'explorer certaines phases décisives des mouvements de musculation comme le contre-mouvement ou encore les projections de charge [14, 28, 49] (figure 5). L'analyse de courbe apparaît également intéressante pour mettre en évidence les effets très spécifiques de l'entraînement [30]. Ce type d'analyse permet de nuancer certaines interprétations liées aux paramètres chiffrés.

\section{Les catégories de mouvements}

Théoriquement, l'évaluation inertielle concerne tout mouvement impliquant le déplacement vertical d'une charge constante. En réalité, l'évaluation inertielle s'adresse avant tout aux mouvement complexes polyarticulaires sollicitant un déplacement linéaire d'une charge (développé couché, squat, presse, 
mouvements haltérophiles, etc.). Ces mouvements globaux se rapprochent des séquences musculaires retrouvées dans les actions sportives et dans les gestes de tous les jours, ce qui leur confère une grande fonctionnalité. Ils ne permettent cependant pas de localiser un déficit de force à l'intérieur d'un mouvement.

L'évaluation musculaire analytique inertielle reste a ce jour quasiment inexplorée. Afin de garantir un déplacement linéaire de la charge, les mouvements devront se réaliser sur des bancs de musculation et non avec des poids et haltères. Bien souvent, les machines ne sont pas adaptées à une utilisation dynamique pourtant nécessaire lors de l'évaluation inertielle. La fin de mouvement, limitée par une butée mécanique ou par la masse musculaire pourrait poser problème à vitesse élevée. Par ailleurs, certains concepts de l'exploration musculaire analytique, retrouvé notamment en isocinétisme (ratio agonistes/antagonistes, ratio mixtes, ...) [11] restent actuellement inaccessibles. Il apparaît évident que la recherche scientifique doit encore démontrer la pertinence de l'évaluation inertielle dans un contexte analytique.

\section{Les modalités d'exécution}

L'évaluation inertielle peut se réaliser selon différentes modalités. Une clarification des avantages et inconvénients, des risques et des recommandations apparaît nécessaire. En effet, les évaluateurs se posent généralement plusieurs questions : le mouvement doit-il être réalisé avec ou sans contre-mouvement? Doit-on inclure une projection de la charge ? L'évaluation excentrique est-elle envisageable ?

L'exécution d'un mouvement complet, qui comprend une phase initiale excentrique suivie immédiatement de la phase concentrique, représente le modèle le plus courant. Les groupes musculaires impliqués subissent donc un 
allongement avant de se raccourcir. Cette modalité d'effort, sollicitant le cycle étirement-détente, apparaît fonctionnelle car la grande majorité des gestes quotidiens (marche, course, etc.) et sportifs (lancers, sauts, etc.) reproduisent ce mécanisme. Le mouvement complet présente néanmoins des inconvénients :

- la performance évaluée ne dépend pas exclusivement du processus contractile, mais englobe la composante élastique musculaire et la capacité individuelle à utiliser ce potentiel ;

- la qualité du contre-mouvement influence fortement la performance et une maîtrise technique apparaît nécessaire pour l'obtention de résultats valides ;

- I'amplitude et la vitesse de la phase excentrique demeurent difficiles à standardiser. Une faible modification à ce niveau pourrait avoir des répercussions élevées sur la performance mesurée [59].

Lorsque l'exploration inertielle doit analyser spécifiquement la performance contractile concentrique, il faut exclure le contre-mouvement. Les conditions d'exécution purement concentriques semblent mieux standardisées. En développé couché par exemple, la barre repose initialement sur des taquets de sécurité, quelques centimètres au-dessus de la poitrine $[14,25,29,61]$. En squat, le mouvement débute avec une flexion standardisée des genoux et des hanches (généralement $90^{\circ}$ ). Le mouvement concentrique consiste à réaliser une extension complète segmentaire $[29,55]$.

La comparaison du mouvement complet avec le simple mouvement concentrique permettrait d'obtenir des informations intéressantes sur la capacité à utiliser avec efficacité le cycle étirement - détente. Ainsi, de nombreux auteurs ont comparé 
la différence entre le squat jump et le contre-mouvement jump, afin de définir les qualités élastiques individuelles [8]. Certains travaux se sont plus récemment intéressés au développé couché $[14,28,49,67]$.

Lors de nombreux gestes quotidiens ou sportifs, l'effort musculaire aboutit à une projection ou à une auto-projection. Lorsque l'évaluation inertielle vise à explorer la composante musculaire dynamique, il peut s'avérer judicieux de réaliser le mouvement avec une projection de la charge. C'est ce qui se passe lorsque l'on réalise un squat jump ou lorsque l'on lance la barre vers le haut lors d'un développé couché. L'intensité du mouvement est alors maximale jusqu'au bout du mouvement, comme lors d'un saut ou d'un lancer [13, 28, 48]. L'effet bénéfique se réduirait avec la charge et, à partir de $60 \%$ du $1 \mathrm{RM}$, il devient inutile de projeter la barre [13]. Il est évident que si l'évaluation avec projection semble avantageuse dans de nombreux contextes, elle ne peut s'envisager que dans des conditions de sécurité optimales. En effet, sans matériel sophistiqué, toute projection de charge s'accompagne d'une réception excentrique très intense, potentiellement dangereuse. Pour les mouvements libres, il est conseillé d'utiliser un guide barre et des taquets de sécurité. Certaines machines sophistiquées retiennent la charge en position haute afin d'éviter une réception potentiellement dangereuse $[14,43,49,54,67]$. Cette technologie demeure cependant onéreuse et, dans la plupart des cas, il reste conseillé de ne réaliser la projection qu'au moyen de charges très faibles et chez des sujets avertis. Par ailleurs, les mouvements avec projection restent difficilement réalisables sur les bancs de musculation classiques qui ne sont généralement pas conçus pour subir un travail hyper dynamique. 
L'évaluation inertielle excentrique existe mais de réalisation difficile et nécessitant une technologie de pointe [43-44]. L'effort, d'intensité maximale, consiste à résister à une charge supra-maximale sur une amplitude de mouvement définie. Murphy et al. [43] proposent, pour le développé couché, un effort excentrique réalisé contre une charge de 130 ou $150 \%$ du $1 \mathrm{RM}$ concentrique entre 120 et $90^{\circ}$ de flexion des coudes. Pour le squat, les mêmes auteurs suggèrent d'utiliser une charge correspondant à $200 \%$ du poids corporel [44]. Le concept, bien qu'intéressant, comporte un risque de blessure significatif, les conditions de sécurité n'étant pas aussi bien développées que pour le dynamomètre isocinétique.

\section{Importance de l'instruction}

L'instruction donnée au sujet avant l'effort pourrait influencer le résultat [5]. Elle doit donc être rigoureusement définie. Certains auteurs donnent comme consigne de développer la vitesse la plus élevée dès le début du mouvement [54, 61]. Cette consigne semble adéquate pour les disciplines sportives qui requièrent un développement très élevé de force et de puissance dès le début du mouvement, comme en boxe, ou comme dans un départ en starting-block par exemple. Cette procédure pourrait s'avérer dangereuse lorsque la charge augmente $[31,62]$. Un développement trop impulsif de force, avec une charge presque maximale pourrait favoriser la survenue de blessures musculaires. Lors d'exercices sollicitants comme le squat, dans le cadre de populations inexpérimentées, il est plutôt conseillé - aux charges les plus élevées - de développer l'accélération jusqu'à la fin du mouvement. Dans tous les cas, le sujet devra, au moment de l'effort, être directement en contact avec la résistance. Nous conseillons même de réaliser une pré-contraction musculaire initiale. 
Lorsque le mouvement s'accompagne d'une projection ou d'un saut, la consigne pourra être simplement d'atteindre la hauteur la plus élevée possible.

\section{Reproductibilité et sensibilité de l'évaluation inertielle}

La reproductibilité de l'évaluation inertielle, relativement bonne dans son ensemble, dépend de nombreux facteurs comme le mouvement, la modalité d'effort, le paramètre, la charge et l'expérience du sujet $[21,30,43,64]$. Le tableau 3 offre un aperçu des coefficients de variation et de corrélation relevés dans la littérature. Les études restent difficilement comparables car ces coefficients sont souvent obtenus par des calculs différents.

La reproductibilité se réduirait avec l'augmentation de la complexité du mouvement [21]. Un mouvement analytique, mono-articulaire est plus simple à apprendre et à reproduire qu'un mouvement complexe poly-articulaire. Ceci est particulièrement vrai pour les exercices comme le squat et le développé couché qui sollicitent au moins trois articulations. Une erreur d'exécution peut se répercuter directement sur la performance et altérer la reproductibilité de l'évaluation. Pour cette raison, il est indispensable d'une part que le sujet soit bien familiarisé avec le mouvement, et d'autre part, d'accorder plusieurs tentatives à chaque niveau de charge afin de conserver la meilleure.

Tableau 3 - Reproductibilité de l'évaluation inertielle

\begin{tabular}{llllll}
\hline Références & Mouvements & Charge(s) & Paramètres & $\begin{array}{c}\text { Coefficient de } \\
\text { variation (CV\% ) }\end{array}$ & $\begin{array}{c}\text { Coefficient de } \\
\text { corrélation (r) }\end{array}$ \\
\hline $\begin{array}{l}\text { Abernethy et al } \\
{[3]}\end{array}$ & Tous & maximale & 1 RM & & {$[0,92-0,98]$} \\
\hline Bosco et al. [7] & SQ & $100 \%$ PC & Dmax, Pmoy, 3,2\% [1,4-5,2\%] & {$[0,85-0,97]$} \\
& & Vmoy, Fmoy, & \\
\hline
\end{tabular}




\begin{tabular}{|c|c|c|c|c|c|c|}
\hline $\begin{array}{l}\text { Funato et al. } \\
{[18]}\end{array}$ & $\begin{array}{l}\text { Rétrop bras, } \\
\text { Flex/Ext } \\
\text { Hanche, Ext } \\
\text { genou, Flexion } \\
\text { coude, SQ, } \\
\text { Tirage, rowing, }\end{array}$ & $\begin{array}{l}42,5 \mathrm{~N} \\
90,6 \mathrm{~N} \\
261,5 \mathrm{~N}\end{array}$ & $\begin{array}{l}\text { Pmax, } \\
\text { F à Pmax } \\
\text { V à Pmax }\end{array}$ & & $4,3 \%[0,2-10,5 \%]$ & {$[0,69-0,97]$} \\
\hline $\begin{array}{ll}\text { Hortobagyi } & \text { et } \\
\text { Katch [22] } & \end{array}$ & SQ, DC & $\begin{array}{l}20 \mathrm{~kg}(\mathrm{DC}) \\
70 \mathrm{~kg}(\mathrm{SQ})\end{array}$ & $\begin{array}{l}\text { Vmax, } \\
\text { Pmoy, } \\
\text { Temps }\end{array}$ & $\begin{array}{r}\text { Pmax, } \\
\text { Tpmax, }\end{array}$ & $?$ & {$[0,77-0,97]$} \\
\hline $\begin{array}{l}\text { Richards et al. } \\
{[56]}\end{array}$ & $\begin{array}{l}\text { Flex/Ext Genou } \\
\text { RI/RE épaule }\end{array}$ & $\begin{array}{l}20-60 N \\
10-20 N\end{array}$ & $\begin{array}{l}\text { Dmax } \\
\text { Vmax } \\
\text { MFM }\end{array}$ & & $?$ & {$[0,73-0,94]$} \\
\hline $\begin{array}{l}\text { Wilson et al. } \\
{[67]}\end{array}$ & DC & $30-60 \% 1 R M$ & $\begin{array}{l}\text { Vmax } \\
\text { W sur } 370\end{array}$ & $\mathrm{~ms}$ & $4,3 \%[1,8-7,6 \%]$ & $?$ \\
\hline $\begin{array}{l}\text { Rahmani et al. } \\
{[55]}\end{array}$ & SQ & $\begin{array}{l}60-80-100-120-140-160- \\
180 \mathrm{~kg}\end{array}$ & $\begin{array}{l}\text { Fmax, } \\
\text { Pmax }\end{array}$ & Vmax, & $4 \%[1,7-6,7 \%]$ & {$[0,70-0,91]$} \\
\hline $\begin{array}{l}\text { Murphy et al. } \\
{[45]}\end{array}$ & DC & $30-60-90-100-130 \%$ 1RM & Fmax & & $?$ & {$[0,90-0,95]$} \\
\hline $\begin{array}{l}\text { Jidovtseff et al. } \\
\text { [29] }\end{array}$ & $\mathrm{DC}, \mathrm{SQ}$ & $\begin{array}{l}35-50-70-90 \% \text { 1RM (DC) } \\
45-60-75-90 \% \text { 1RM (SQ) }\end{array}$ & $\begin{array}{l}\text { Vmax, } \\
\text { Pmax, } \\
\text { Tpmax }\end{array}$ & $\begin{array}{l}\text { Vmoy, } \\
\text { Pmoy, }\end{array}$ & $7 \%[2,5-16,3 \%]$ & $?$ \\
\hline $\begin{array}{l}\text { Viitasalo et al. } \\
{[63]}\end{array}$ & SQ jump & $0-20-40-60-80 \mathrm{~kg}$ & Fmax & & $6,5 \%[4,3-9,5 \%]$ & $?$ \\
\hline Newton [49] & SQ jump & $30-60-90 \% 1 R M$ & $\begin{array}{l}\text { Dmax, } \\
\text { Vmax, } \\
\text { Fmoy, } \\
\text { Pmoy }\end{array}$ & $\begin{array}{l}\text { Pmax, } \\
\text { Fmax, }\end{array}$ & $?$ & {$[0,69-0,99]$} \\
\hline McCurdy [42] & SQ 1jambe & maximale & 1RM, 3RM & & & {$[0,87-0,99]$} \\
\hline
\end{tabular}

La standardisation des mouvements réduit les facteurs de variabilité. Dans certains cas, le seul mouvement concentrique apparaît plus reproductible que le mouvement complet ; en effet, le contre mouvement reste difficile à contrôler pour un sujet non initié. La vitesse de la phase excentrique et son amplitude constituent deux éléments non contrôlables qui perturbent la performance musculaire et qui peuvent être source d'une plus grande variabilité. Une exécution incorrecte de cette phase doit conduire à l'annulation de l'essai et 
parfois, une accumulation des répétitions peut engendrer une fatigue musculaire non négligeable [30].

La reproductibilité de l'évaluation inertielle s'altérerait lorsque la charge devient élevée et le mouvement lent [29, 43, 64]. En effet, aux charges les plus élevée, la difficulté à mobiliser une charge très lourde dans les premiers centimètres explique parfois un temps d'effort très variable et une plus faible reproductibilité des paramètres inertiels, surtout moyens. Aux charges très légères, la reproductibilité dépend des conditions de test $[22,30]$. Chez certains sujets débutants, l'action musculaire frénatrice de fin de mouvement apparaît inconstante, altérant la reproductibilité.

La reproductibilité varie selon le paramètre étudié. L'accumulation des étapes mathématiques nécessaires pour obtenir un paramètre affecte inévitablement sa variabilité. La vitesse qui ne fait I'objet que d'une seule opération mathématique apparaît ainsi plus reproductible que la puissance qui fait l'objet de deux opérations mathématiques. Plusieurs études confirment la plus grande variabilité de la puissance, non seulement en évaluation inertielle, mais aussi isocinétique $[7,27,55,57]$. Les paramètres liés au temps (Tpmax et Tvmax) présentent généralement une reproductibilité plus inconstante, surtout à charge élevée.

Les quelques études ayant exploré la sensibilité de l'évaluation dynamométrique inertielle présentent des résultats encourageants $[2,7,30,43-45,49]$. Cette technique moderne permettrait une évaluation sensible de la fonction musculaire fournissant des informations judicieuses sur les qualités de force-vitessepuissance et d'endurance locale. Des travaux récents montrent qu'elle serait 
discriminante entre les individus et selon la discipline sportive $[7,26,30]$. La diversité des profils charge-vitesse confirme que les sujets les plus forts ne sont pas nécessairement les plus véloces et les sujets les plus véloces ne sont pas toujours les plus forts [30]. L'évaluation inertielle semble rassembler les qualités nécessaires pour devenir une technique de référence lorsque l'on souhaite objectiver les effets spécifiques d'un entraînement musculaire. Les mouvements tests correspondant à ceux de l'entraînement, la spécificité et la sensibilité de l'évaluation sembleraient optimaux. Cette spécificité peut cependant parfois apparaître comme un désavantage. En effet, les gains observés pour certains mouvements complexes répondent en partie d'un apprentissage technique, qui ne pourra jamais être dissocié des vrais gains musculaires. Les utilisateurs doivent rester conscients du fait que ces tests permettent d'objectiver des améliorations dans les gestes de musculation et non pas dans les gestes fonctionnels [44]. Il est évident que le transfert des gains sera d'autant plus important que les gestes se ressemblent. Un défi de la recherche actuelle est d'établir les liens et les transferts qui existent entre les améliorations de la fonction musculaire et les améliorations fonctionnelles dans le geste sportif ou dans les actions de la vie de tous les jours.

\section{Conclusions et perspectives}

L'évaluation inertielle possède l'avantage incontestable de respecter un modèle de contraction musculaire naturel, impliquant l'accélération et la décélération d'une masse constante. Cette cinétique gestuelle, sous l'influence de la gravité, reproduit ce qui se passe dans la majorité des gestes sportifs et dans de nombreux mouvements quotidiens. 
L'évaluation inertielle reste possible avec peu de matériel, mais l'approche dynamométrique apparaît beaucoup plus intéressante en autorisant l'appréciation des qualités de vitesse et de puissance à des charges sous-maximales. Contrairement à d'autres techniques, l'évaluation dynamométrique inertielle est transportable et peut s'envisager sur le lieu même de l'entraînement. Elle devrait permettre une exploration exhaustive des relations charge-vitesse et chargepuissance puisque, théoriquement, ni la charge, ni la vitesse ne sont limitées. Cette possibilité d'exprimer une vitesse réellement maximale pour une charge donnée constitue, sans aucun doute, un avantage majeur de la méthode inertielle. En pratique, l'évaluation inertielle peut être limitée par le matériel et par les conditions de sécurité. La perspective de réaliser une évaluation avec projection apparaît particulièrement intéressante, mais ne peut s'envisager que sous certaines conditions. Les dynamomètres inertiels offrent, par ailleurs, des perspectives de recherches dans l'exploration de la fatigue musculaire ou encore dans l'analyse biomécanique des mouvements.

L'évaluation inertielle s'avère reproductible pour la majorité des paramètres. Elle apparaît également sensible. Son caractère discriminant permet de comparer des profils musculaires et de situer un individu à l'intérieur d'un groupe. Elle apparaît particulièrement adaptée pour apprécier les effets d'un entraînement composé d'exercices de poids et haltères.

Cependant, les protocoles rencontrés varient énormément dans la littérature. Leur uniformisation, mais aussi une clarification des recommandations s'avèrent indispensables à la connaissance et à la validation de cette technique d'évaluation. 
Actuellement, l'évaluation inertielle concerne principalement des mouvements globaux, s'accompagnant d'un déplacement linéaire de la charge. L'exploration musculaire analytique reste par contre peu étudiée. Une adaptation des bancs de musculation pourrait s'envisager dans cette perspective de recherche. Il reste à vérifier si l'approche inertielle pourrait offrir, dans ce contexte analytique, des informations aussi pertinentes que l'isocinétisme en termes d'équilibre musculaire et de survenue lésionnelle.

Les applications dans le domaine sportif apparaissent nombreuses et devront susciter l'imagination des scientifiques et praticiens. Il apparaît clairement que l'évaluation dynamométrique inertielle est une technique d'avenir qui devrait aboutir à une utilisation plus fréquence, mais qui doit rester rigoureuse.

\section{Références}

1. Abadie BR, Wentworth MC. Prediction of one repetition maximal strength from a 5-10 repetition submaximal strength test in college-aged females. J Exerc Physiol 2000; 4: 1-6.

2. Abernethy $P$, Jürimäe J. Cross-sectional and longitudinal uses of isoinertial, isometric, and isokinetic dynamometry. Med Sci Sports Exerc 1996; 28: 1180-7.

3. Abernethy $\mathrm{P}$, Wilson G, Logan P. Strength and power assessment. Issues, controversies and challenges. Sports Med 1995; 19: 401-17.

4. Almasbakk B, Hoff J. Coordination, the determinant of velocity specificity? J Appl Physiol 1996; 81: 2046-52.

5. Behm DG, Sale DG. Intended rather than actual movement velocity determines velocity-specific training response. J Appl Physiol 1993; 74: 359-68. 
6. Berger RA. Optimum repetitions for the development of strength. Res Q 1961; 33: 334-8.

7. Bosco C, Belli A, Astrua M, Tihanyi J, Pozzo R, Kellis S, Tsarpela O, Foti C, Manno R, Tranquilli C. A dynamometer for evaluation of dynamic muscle work. Eur J Appl Physiol 1995 ; 70: 379-86.

8. Bosco C. Evaluation de la force par le test de Bosco. Rome: Stampa Sportiva; 1992.

9. Brown L, Weir J. ASEP procedures recommendation I: Accurate assessment of muscular strength and power. J Ex Physiol 2001; 4: 1-21.

10. Canavan PK, Vescovi JD. Evaluation of power prediction equations: peak vertical jumping power in women. Med Sci Sports Exerc 2004; 36: 1589-93.

11. Croisier JL, Forthomme B, Namurois MH, Vanderthommen M, Crielaard JM. Hamstring muscle strain recurrence and strength performance disorders. Am J Sports Med 2002 ; 30: 199-203.

12. Cronin J, Sleivert G. Challenge in understanding the training influence of maximal power training on improving athletic performance. Sports Med 2005; 35: 213-234.

13. Cronin JB, McNair PJ, Marshall RN. Force-velocity analysis of strength-training techniques and load: implications for training strategy and research. J Strength Cond Res 2003; 17: 148-55.

14. Cronin JB, McNair PJ, Marshall RN. Magnitude and decay of stretch-induced enhancement of power output. Eur J Appl Physiol 2001; 84: 575-581.

15. Dempsey PG, Ayoub MM, Westfall PH. Evaluation of the ability of power to predict low frequency lifting capacity. Ergonomics 1998; 41: 1222-1241.

16. Epley B. Poundage chart. Boyd Epley workout. Nebraska: Lincoln; 1985.

17. Escamilla RF, Fleisig GS, Zheng N, Barrentine SW, Wilk KE, Andrews JR. Biomechanics of the knee during closed kinetics chain and open kinetic chain exercises. Med Sci Sports Exerc 1998; 30: 556-69.

18. Funato K, Matsuo A, Fukunaga T. Measurement of specific movement power application: evaluation of weight lifters. Ergonomics 2000; 43: 40-54. 
19. Häkkinen K, Pakarinen A, Alen M, Kauhanen H, Komi PV. Neuromuscular and hormonal adaptations in athletes to strength training in two years. J Appl Physiol 1988; 65: 2406-12.

20. Harman EA. The measurement of human mechanical power. In: Maud PJ, Foster C. editors. Physiological assessment of human fitness. Human Kinetics Champaign; 1995. p. 87-113.

21. Hopkins WG, Schabort EJ, Hawley JA. Reliability of power in physical performance tests. Sports Med 2001; 31: 211-34.

22. Hortobagyi T, Katch F. Reliability of muscle mechanical characteristics for isokinetic and isotonic squat and bench press exercise using a multifunction computerized dynamometer. Res Quat Exerc Sports 1990; 61: 191-5.

23. Horvat M, Ramsey V, Franklin C, Gavin C, Palumbo T, Glass LA. A method for predicting maximal strength in collegiate women athletes. J Strength Cond Res 2003; 17: 324-8.

24. Inbar O, Bar-Or O, Skinner JS. The wingate anaerobic test. Human Kinetics Champaign; 1996.

25. Izquierdo M, Ibanez J, Gonzalez-Badillo JJ, Gorostiaga EM. Effects of creatine supplementation on muscle power, endurance, and sprint performance. Med Sci Sports Exerc 2002; 34: 332-43.

26. Izquierdo M, Ibanez J, Gorostiaga E, Garrues M, Zuniga A, Anton A, Larrion JL, Häkkinen K. Maximal strength and power characteristics in isometric and dynamic actions of the upper and lower extremities in middle-aged and old men. Acta Physiol Scand 1999; 167: 57-68.

27. Jablonowsky R, Inbar O, Rotstein A, Tenenbaum G. Evaluation of anaerobic performance capacity by the isokinetics ariel computerized exercise systemreliability and validity. J Sports Med Phys Fitness 1992; 32: 262-70.

28. Jidovtseff B, Croisier JL, Crielaard, JM. Influence de la modalité du développé couché sur la performance iso-inertielle. Sci Sports 2006; 21: 159-62. 
29. Jidovtseff B, Croisier JL, Lhermerout C, Serre L, Sac D, Crielaard JM. The concept of iso-inertial assessment: reproducibility analysis and descriptive data. Isokinetics Exerc Sci 2006 ; 14: 53-62.

30. Jidovtseff $B$. Mise au point d'un dynamomètre de la puissance musculaire.[Thèse de Doctorat]. Liège (Belgique) : Université de Liège; 2006.

31. Kazarian L. Injuries to the human spinal column: biomechanics and injury classification. In: Miller DI editor. Exerc Sports Sci Rev Philadelphia; 1981. p. 297-352.

32. Kraemer WJ, Fry AC. Strength testing: development and evaluation of methodology. In : Maud PJ, Foster C editors. Physiological assessment of human fitness. Human Kinetics Champaign; 1995. p. 115-38

33. Kroll W. Reliability of a selected measure of human strength. Res Q Exerc Sport 1961; 33: 410-17.

34. Lander J. Maximum based on reps. Nat Strength Conditioning Assoc J 1985; 6: $60-1$.

35. Lehance C, Croisier JL, Bury T. Validation du système Optojump en tant qu'outil d'évaluation de la force-vitesse (puissance) des membres inférieurs. Sci Sports 2005; 20: 131-5.

36. Logan P, Fornasiero D, Abernethy P, Lynch K. Protocols for the assessment of isoinertial strength. In : Gore CJ ed. Physiological tests for elites athletes. Human Kinetics Champaign ; 2000. p. 200-221

37. Lombardi VP. Beginning Weight Training: The Safe and Effective Way. Brown Company Publishers 1989.

38. Lygren $\mathrm{H}$, Dragesund $\mathrm{T}$, Joensen J, Ask T, Moe-Nilssen R. Test-retest reliability of the progressive isoinertial lifting evaluation (PILE). Spine 2005; 30: 1070-4.

39. Madsen N, McLaughlin T. Kinematic factor influencing performance and injury risk in the bench press exercise. Med Sci Sports Exerc 1984; 16: 376-81.

40. Mayhew JL, Ball TE, Arnold MD, Bowen JC. Relative muscular endurance performance as a predictor of bench press strength in college men and women. J Appl Sport Sci Res 1992; 6: 200-6. 
41. Mayhew JL, Prinster JL, Ware JS, Zimmer DL, Arabas JR, Bemben MG. Muscular endurance repetitions to predict bench press strength in men of different training levels. J Sports Med Phys Fitness 1995; 35: 108-13.

42. McCurdy K, Langford GA, Cline AL, Doscher M, Hoff R. The reliability of 1- and 3RM tests of unilateral strength in trained and untrained men and women. $\mathrm{J}$ Sports Sci Med 2004; 3: 190-6.

43. Murphy AJ, Wilson GJ, Pryor JF. Use of the iso-inertial force mass relationship in the prediction of dynamic human performance. Eur J Appl Physiol 1994; 69: 250-7.

44. Murphy AJ, Wilson GJ. The ability of tests of muscular function to reflect traininginduced changes in performance. J Sports Sci 1997; 15: 191-200.

45. Murphy AJ, Wilson GJ. The assessment of human dynamic muscular function: $A$ comparison of isoinertial and isokinetic tests. J Sports Med Phys Fitness 1996; 36: $169-77$.

46. Newton RU, Häkkinen K, Häkkinen A, Mccormick M, Volek J, Kraemer W. Mixedmethods resistance training increase power and strength of young and older men. Med Sci Sports Exerc 2002; 34: 1367-75.

47. Newton RU, Kraemer W, Häkkinen K. Effects of ballistic training on preseason preparation of elite volleyball players. Med Sci Sports Exerc 1999; 31: 323-30.

48. Newton RU, Kraemer WJ, Häkkinen K, Humphries BJ, Murphy AJ. Kinematics, kinetics and muscle activation during explosive upper body movements. J Appl Biomech 1996; 12: 31-43.

49. Newton RU. Expression and development of maximal muscle power. [Thèse de Doctorat]. Queensland (Australie) : University of Queensland; 1997.

50. O'Conner B, Simmons J, O'Shea P. Weight training today. West pubishing; 1989. p. 26-33.

51. Parcell AC, Sawyer RD, Tricoli VA, Chinevere TD. Minimum rest period for strength recovery during a common isokinetic testing protocol. Med Sci Sports Exerc 2002; 34: 1018-22. 
52. Pereira MI, Gomes PS. Muscular strength and endurance tests: reliability and prediction of one repetition maximum. Review and new evidence. Rev Bras Med Esporte 2003; 9: 336-46,.

53. Poortmans JR, Boisseau N. Biochimie des activités physiques. Bruxelles: DeBoeck Université ; 2002.

54. Pryor JF, Wilson GJ, Murphy AJ. The effectiveness of eccentric, concentric and isometric rate of force development tests. J Human Mov Studies 1994; 27: 15372.

55. Rahmani A, Dalleau G, Viale F, Hautier C, Lacour JR. Validity and reliability of kinematic device for measuring the force developed during squatting. J Appl Biomech 2000; 16: 26-35.

56. Richards JG, Quigley EJ, Castagno PW, Neeves RE. Validity and reliability of the BTE Dynatrac. Med Sci Sports Exerc 1996; 28: 913-20.

57. Sale DG. Testing strength and power. In : MacDougall JD, Wenger HA, Green $\mathrm{HJ}$, editors. Physiological testing of the high-performance athlete. Human Kinetics Champaign; 1991. p. 21-103.

58. Stauber WT, Barill ER, Stauber RE, Miller GR. Isotonic dynamometry for assessment of power and fatigue in the knee extensor muscles of females. Clin Physiol 2000; 20: 225-33.

59. Takarada $\mathrm{Y}$, Hirano $\mathrm{Y}$, Ishige $\mathrm{Y}$, Ishii N. Stretch-induced enhancement of mechanical power output in human multijoint exercise with countermovement. J Appl Physiol 1997; 83: 1749-55.

60. Thomas M, Fiatarone MA, Fielding RA. Leg power in young women: relationship to body composition, strength, and function. Med Sci Sports Exerc 1996; 28: $1321-6$

61. Thompson CJ, Bemben MG. Reliability and comparability of the accelerometer as a measure of muscular power. Med Sci Sports Exerc 1999; 31: 897-902.

62. Verdera F, Champavier L, Schmidt C, Bermon S, Marconnet P. Reliability and validity of a new device to measure isometric strength in polyarticular exercises. J Sports Med Phys Fitness 1999; 39: 113-9. 
63. Viitasalo JT, Häkkinen K, Komi PV. Isometric and dynamic force production and muscle fibre composition in man. J Human Movement Stud 1981; 7: 199-209.

64. Viitasalo JT. Evaluation of explosive strength for young and adult athletes. Res Q Exerc Sport 1988; 59: 9-13.

65. Viitasalo JT. Measurement of force-velocity characteristics for sportsmen in field conditions. In: Winter DA, Norman RM, Wells RP, et al., eds. Biomechanics IX-A. Human Kinetics Champaign; 1985. p. 96-101.

66. Vøllestad NK. Measurement of human muscle fatigue. J Neurosci Methods 1997; 74: 219-27.

67. Wilson GJ, Murphy AJ, Giorgi A. Weight and plyometric training: effects on eccentric and concentric force production. Can J Appl Physiol 1996; 21: 301-15.

68. Wilson GJ, Newton RU, Murphy AJ, Humphries BJ. The optimal training load for the development of dynamic athletic performance. Med Sci Sports Exerc 1993; 25: $1279-86$.

69. Wilson GJ, Walshe AD, Fischer MR. The development of an isokinetic squat device: reliability and relationship to functional performance. Eur J Appl Physiol 1997; 75: 455-61.

70. Wilson GJ. Strength and power assessment. In: Bloomfield, Ackland et Elliott editors. Applied anatomy and Biomechanics in sport. Blackwell Science Asia; 1994.

71. Wrigley T., Strauss G. Strength assessment by isokinetics dynamometry. In: Gore C.J. editor. Physiological tests for elite athletes. Human Kinetics Champaign; 2000. p. 155-99. 